\title{
Astrocytic histone deacetylase 2 facilitates delayed depression and memory impairment after subarachnoid hemorrhage by negatively regulating glutamate transporter-1
}

\author{
Kai Tao ${ }^{1 \#}$, Qing Cai ${ }^{1 \#}$, Xudong Zhang ${ }^{2 \#}$, Lin Zhu ${ }^{1}$, Zhenru Liu ${ }^{3}$, Fei Li $^{1}$, Qiang Wang ${ }^{1}$, Lei Liu ${ }^{4}$, \\ Dayun Feng ${ }^{1,3}$
}

${ }^{1}$ Department of Neurosurgery and Institute for Functional Brain Disorders, Tangdu Hospital, Fourth Military Medical University, Xi'an, China; ${ }^{2}$ China-Nepal Friendship Medical Research Center of Rajiv Kumar Jha, School of Clinical Medicine, Xi'an Medical University, Xi'an, China; ${ }^{3}$ School of Basic Medicine, Fourth Military Medical University, Xi'an, China; ${ }^{4}$ Department of Gastroenterology, Tangdu Hospital, Fourth Military Medical University, Xi'an, China

Contributions: (I) Conception and design: D Feng, L Liu; (II) Administrative support: L Liu; (III) Provision of study materials: F Li, Q Wang; (IV) Collection and assembly of data: K Tao, Q Cai, Z Liu; (V) Data analysis and interpretation: X Zhang, L Zhu; (VI) Manuscript writing: All authors; (VII) Final approval of manuscript: All authors.

\#These authors contributed equally to this work.

Correspondence to: Dayun Feng, MD, PhD. Department of Neurosurgery and Institute for Functional Brain Disorders, Tangdu Hospital, Fourth Military Medical University, No. 569 Xinsi Road, Xi’an, China; Email: tdfengdy@fmmu.edu.cn; Lei Liu, MD, PhD. Department of Gastroenterology, Tangdu Hospital, Fourth Military Medical University, No. 569 Xinsi Road, Xi'an, China; Email: tdliulei@fmmu.edu.cn.

Background: Delayed cognitive impairment (DCI) after subarachnoid hemorrhage (SAH) is one of the most common sequelae in patients. This study aimed to investigate the characteristics of the course and glutamatergic pathogenesis of DCI after SAH in mice.

Methods: A SAH mouse model of internal carotid puncture was used. Depressive and cognitive behaviors were detected by forced swimming and sucrose preference tests and Morris water maze test, respectively. Microdialysis and high-performance liquid chromatography (HPLC) were used to detect the interstitial glutamate. The expressions of histone deacetylases (HDACs), glutamate transporters, and glutamate receptors were examined. Primary astrocytes magnetically sorted from adult mice were cultured for glutamate uptake assay and protein and mRNA detection. Selective HDAC2 inhibitor and glutamate transporter-1 (GLT-1) inhibitor administered via were intraperitoneal injection to evaluate their effects on DCI in SAH mice.

Results: Depression and memory impairment lasted for more than 12 weeks and peaked at 8 weeks after SAH. Interstitial glutamate accumulation in the hippocampus and impaired glutamate uptake in astrocytes of the SAH mice were found during DCI, which could be explained by there being a significant decrease in GLT-1 expression but not in glutamate and aspartate transporter (GLAST) in hippocampal astrocytes. Meanwhile, the phosphorylation level of excitatory glutamate receptors (GluN2B and GluA1) in the hippocampus was significantly reduced, although there was no significant change in the expression of the receptors. Importantly, the expression of HDAC2 increased most significantly in astrocytes after SAH compared with that of other subtypes of HDACs. Inhibition of HDAC2 markedly rescued the decrease in GLT-1 expression after SAH through transcriptional regulation. Behavioral results showed that a selective HDAC2 inhibitor effectively improved DCI in SAH mice, but this effect could be weakened by GLT-1 inhibition.

Conclusions: In summary, our study suggests that the dysfunction of GLT-1-mediated glutamate uptake in astrocytes may be a key pathological mechanism of DCI after SAH, and that a specific inhibitor of HDAC2 may exert a potential therapy. 
Keywords: Subarachnoid hemorrhage (SAH); delayed cognitive impairment (DCI); astrocyte; GLT-1; HDAC2

Submitted May 13, 2020. Accepted for publication Jun 09, 2020.

doi: $10.21037 /$ atm-20-4330

View this article at: http://dx.doi.org/10.21037/atm-20-4330

\section{Introduction}

Spontaneous subarachnoid hemorrhage (SAH), one of the most common hemorrhagic cerebrovascular diseases, is principally caused by ruptured intracranial aneurysms (1). Severe cognitive impairment, including depression, anxiety, and memory impairment, affects more than $20 \%$ of patients who survive (2). The majority of survive SAH patients develop depressive symptoms two weeks after onset, and after three months, the effects of memory impairment are still apparent. While acute neurological deficiency is caused by damage and death of nerve cells, chronic persistent cognition may be related to impaired hippocampal capacity and neural plasticity (3). However, the pathological characteristics and cellular and molecular mechanisms of DCI after SAH are not have yet to be fully illuminated.

In cognitive and psychological diseases, the disturbance of the neurotransmitter system, especially the excitatory glutamate transmitter system, has recently attracted a considerable amount of attention (4). Various studies have demonstrated an abnormal increase in glutamate levels in cerebrospinal fluid (CSF) and peripheral plasma after stroke, which indicates that the abnormality of glutamatergic transmission may be an important mechanism of secondary DCI after SAH $(5,6)$. Astrocytic glutamate transporters, which are also known as excitatory amino acid transporters (EAATs), serve a vital role in glutamate homeostasis in the central nervous system (CNS). Previous studies have confirmed that GLT-1 (EAAT2), which is mainly expressed in the prefrontal lobe and hippocampus, is downregulated in a range of nerve injury diseases, including SAH, epilepsy, Alzheimer's disease, and posttraumatic stress disorder (7-9). Therefore, further investigation is needed into the relationship between the expression of EAATs and the occurrence of DCI after SAH, as well as the mechanism underlying this process.

While EAATs are regulated by a group of transcription factors, including nuclear transcription factor- $\kappa \mathrm{B}(\mathrm{NF}-$ $\kappa \mathrm{B})$, cAMP-response element binding protein (CREB), and epidermal growth factor (EGF) (10), the inhibition of EAAT gene transcription in chronic pathological processes is also related to histone modification, especially histone acetylation. In neurodegenerative disease, trichostatin A, a broad-spectrum inhibitor of HDAC, has been reported to upregulate the level of GLT-1 (11). However, the changes to HDACs after SAH and their effects on the transcriptional expression of EAATs are not clear, nor is their role in the pathogenesis of DCI after SAH known. To elucidate this, this study aimed to explore the change of HDACs and GLT-1 in SAH and attempt to intervene the behavioral of SAH by applying specific HDACs inhibitor. And this may provide a potential therapy for secondary cognitive impairment after $\mathrm{SAH}$ or other related diseases.

We present the following article in accordance with the ARRIVE reporting checklist (available at http://dx.doi. org/10.21037/atm-20-4330).

\section{Methods}

\section{Animal preparation}

Eighty-six adult C57BL/6 mice (weight: 20.0-25.0 g) were obtained from the Animal Experimental Center of the Fourth Military Medical University. The room temperature was set at $25.0 \pm 3.0^{\circ} \mathrm{C}$, and the $12: 12$ light/dark circadian rhythm was controlled. All animal experiments were scheduled to be carried out in the morning. All procedures were approved by the Institutional Animal Care and Use Committee and performed in accordance with the ethical guidelines of the Fourth Military Medical University (animal approval number: TDLL-2015034). The operations were performed to minimize the number and suffering of animals used.

\section{SAH mouse model and drug administration}

The endovascular perforation model of SAH in mice was performed as previously described (12). Briefly, the mice were anesthetized with $2 \%$ isoflurane. A median incision was made in the neck to expose the left carotid artery bifurcation. The external carotid artery was ligated and dissected, and 6-0 pointed monofilaments were inserted into the internal carotid artery. The puncture breakthrough of internal carotid artery was received when 10-12 mm was inserted to reach the bifurcation of anterior cerebral 
artery and middle cerebral artery, and another $3 \mathrm{~mm}$ was penetrated inward. Then the suture was withdrawal after remaining in place for $20 \mathrm{~s}$. The sham mice underwent the same operation without puncturing the artery vessel. The core temperature of the mice was maintained at $37^{\circ} \mathrm{C}$ during operation.

The SAH mice were intraperitoneally administered with HDAC2 inhibitor (Santacruzamate A, Selleck, CAY10683, $2 \mathrm{mg} / \mathrm{kg}$ ), GLT-1 inhibitor (MedChemExpress, WAY$213613,1 \mathrm{mg} / \mathrm{kg}$ ), and vehicle (10\% Dimethyl sulfoxide $(\mathrm{DMSO})+90 \%$ (20\% Sulfobutylether- $\beta$-Cyclodextrin $(\mathrm{SBE}-\beta-\mathrm{CD})$ in saline)) two weeks after surgery once every other day.

\section{Forced swimming test}

A tank [40 cm (height) $\times 26 \mathrm{~cm}$ (diameter)] was filled with water $\left(23 \pm 2{ }^{\circ} \mathrm{C}\right)$ to a height of $33 \mathrm{~cm}$ to prevent the mice from escaping or supporting themselves against the bottom. The mice were then forced to swim for $6 \mathrm{~min}$. The duration of immobility was measured using a SCANET MV40AQ automatic analysis system (MELQUEST, Co., Ltd., Toyama, Japan).

\section{Sucrose preference test}

Mice were housed individually in a quiet animal house without any disruptions. Before the test, the mice were trained to drink the sucrose solution. Two bottles containing pure water and $2 \%$ sucrose solution were prepared for each mouse. The positions of the two bottles were switched every $24 \mathrm{~h}$. After 3 days of habituation, the water/sucrose consumption test was carried out after $17 \mathrm{~h}$ of food and water deprivation. A bottle of pure water and a bottle of $2 \%$ sucrose solution were weighed in advance and then given to the mice. After $24 \mathrm{~h}$, the two bottles were weighed. The consumption of sucrose solution, pure water, and total water was recorded, and the percentage of sucrose consumed was calculated (13).

\section{Morris water maze test}

The Morris water maze test was performed as previously described (14). Briefly, the mice were trained to find a hidden platform (10 $\mathrm{cm}$ in diameter) in a white plastic tank with water (120 cm in diameter). The mice were subjected to 3 trials per day with an intertrial interval of $30 \mathrm{~min}$. For 5 consecutive days, the learning phase of the water maze test was performed, followed by the probe test on day 6 . During the probe test, the mice were given $1 \mathrm{~min}$ to find the removed platform. The target quadrant occupancy and the exact number of crossings over the former platform location during the probe test were measured using the EthoVision 10 program (Noldus, Netherlands).

\section{Interstitial fluid microdialysis}

Microdialysis was used to collect hippocampal interstitial fluid according to the previous study (15). In brief, a probe (4-mm cannula length, 0.22-mm outer diameter, 1.0-mm inter length; Eicom Corp) was stereotaxically inserted into the hippocampus of the mice $(2.2 \mathrm{~mm}$ posterior, $1.6 \mathrm{~mm}$ lateral, and $1.6 \mathrm{~mm}$ ventral). Interstitial fluid was collected after continuous perfusion with artificial cerebrospinal fluid and was subsequently lyophilized.

\section{Glutamate HPLC-MS analysis}

The glutamate concentration in the interstitial fluid was assessed using HPLC (2695 Alliance HPLC, Waters, Massachusetts, USA). A total of $20 \mu \mathrm{L}$ of redissolved interstitial fluid sample was prepared and precolumn derivatized with 2,4-dinitrofluorobenzene for 30 minutes at $60{ }^{\circ} \mathrm{C}$. Dihydrogen phosphate $(50 \mathrm{mM})$ was added to microdialysis to stop the reaction later. And then a UV detector (Waters, Massachusetts, USA) was operated to analyze the glutamate concentrations in the microdialysis samples at an absorbance of $360 \mathrm{~nm}$. The concentrations were calculated using LC solution software (Waters, Massachusetts, USA) on the basis of the standard samples (Sigma-Aldrich, Pennsylvania, USA).

\section{Primary astrocyte separation}

First, whole neural cells were isolated from adult mouse forebrain tissue using the Neural Tissue Dissociation Kit (130-092-628, Miltenyi, Germany). Then, magnetic separation was performed to sort purified astrocytes using an anti-ACSA-1 MicroBead Kit (130-095-825, Miltenyi, Germany). Primary astrocytes were cultured or lysed for the in vitro experiments.

\section{Glutamate uptake assay}

Glutamate uptake was assessed according to the method described in our previous study (16). The sorted 
astrocytes were cultured in $60-\mathrm{mm}$ dishes and incubated with glutamate at $200 \mu \mathrm{M}$. Then, the final glutamate concentration in the supernatant was detected at the indicated time points using a glutamate assay kit (ab83389, Abcam, USA).

\section{Western blotting}

Tissue samples were made into protein samples by RIPA lysis buffer and prepared for protein electrophoresis. After electrophoresis, the gels were transferred to polyvinylidene fluoride (PVDF) membranes, then incubated with the indicated antibodies with gentle shaking overnight at $4{ }^{\circ} \mathrm{C}$. The antibodies used were HDAC1 $(1: 1,000$, Cell Signaling Technology, \#34589), HDAC2 (1:1,000, Cell Signaling Technology, \#57156), HDAC3 (1:1,000, Cell Signaling Technology, \#85057), HDAC4 (1:1,000, Cell Signaling Technology, \#15164), HDAC5 (1:1,000, Cell Signaling Technology, \#20458), HDAC6 (1:1,000, Cell Signaling Technology, \#7558), GLT-1 (1:2,000, Cell Signaling Technology, \#3838), GLAST (1:2,000, Cell Signaling Technology, \#5684), GluN2B (1:1,000, Cell Signaling Technology, \#14544), p-GluN2B (1:1,000, Cell Signaling Technology, \#5355), GluA1 (1:1,000, Cell Signaling Technology, \#13185), p-GluA1 (1:1,000, Cell Signaling Technology, 75574), and $\beta$-actin (1:2,000, Cell Signaling Technology, \#4967). Next, the PVDF membranes were incubated with specific HRP-conjugated secondary antibodies, and the protein bands were visualized using enhanced chemiluminescence (ECL, Bio-Rad, California, USA). ImageJ software (National Institutes of Health, USA) was employed to quantify the band densities.

\section{Quantitative PCR}

Total RNA was isolated with TRIzol (Invitrogen, New York, USA) according to the manufacturer's instructions. A reverse transcription kit (Roche, New York, USA) was used to synthesize cDNA. qPCR was performed by using a LightCycler ${ }^{\circledR}$ 480II Detection System with SYBR green incorporation (Roche, New York, USA). The specific primers used to amplify mouse GLT-1 were as follows: forward, 5'-GGGTGAACAGGCCAAGCTGATGG-3' and reverse, 5'-ATGATGAGGCCCACGATCACGG-3'. The mice GAPDH primer sequence: forward: 5 '- TG ACATCAAGAAGGTGGTGGTGAAGCAG-3 ', 5'-GGTCCACCACCCTGTT-GCTGTAG-3' (17). The relative mRNA levels were calculated with the $2^{-\Delta \Delta C t}$ method.

\section{Immunofluorescence staining}

After anesthesia, saline infusion and paraformaldehyde fixation, coronal brain slices $(30 \mu \mathrm{m})$ of the sham and SAH mice were obtained to detect hippocampal morphology. The slices were then incubated with the primary antibodies at $4{ }^{\circ} \mathrm{C}$ overnight following administrations of $0.3 \%$ TritonX-100 for $30 \mathrm{~min}$ and $0.5 \%$ donkey serum for $1 \mathrm{~h}$. Triple-labeling staining was performed using antibodies as HDAC2 (Cell Signaling Technology, USA, \#5113, 1:200), GFAP (NOVUS, NBP 1-05198, 1:4,000, USA) and GLT-1 (Cell Signaling Technology, \#3838, 1:200, USA). Secondary antibodies, Alexa Fluor488 anti-rabbit IgG, Alexa Fluor Plus 594 Goat anti-Chicken IgY, and Alexa Fluor647 antimouse IgG (Invitrogen, USA), were used at 1:500 dilution. Fluorescence images were captured using a confocal microscopy (A1 Si; Nikon, Japan).

\section{Statistical analysis}

The data were expressed as the mean \pm standard error of the mean (SEM) from at least three independent experiments. Behavioral tests were analyzed by two-way analysis of variance (ANOVA) with group as a between-subject factor and time as a repeated-measure factor. Comparisons between data from two groups were analyzed by one-way ANOVA, followed by Tukey's test to compare all pairs of columns according to the number of independent variables. $\mathrm{P}<0.05$ was considered to show statistical significance. All data graphs were generated using GraphPad Prism 6.01 software (San Diego, USA).

\section{Results}

\section{Characteristics of delayed depression and memory impairment after SAH in mice}

Forced swimming and sugar preference tests were carried out to assess depression, and the Morris water maze test was performed to evaluate spatial reference memory, in the sham and SAH groups before and after modeling. One week before the operation, there was no difference in depressive behavior between the sham group and the SAH group (Figure 1A,B). After the operation, the SAH group showed persistent depressive behavior over time compared with the sham group $(\mathrm{P}<0.01$, two-way ANOVA). The $\mathrm{SAH}$ 

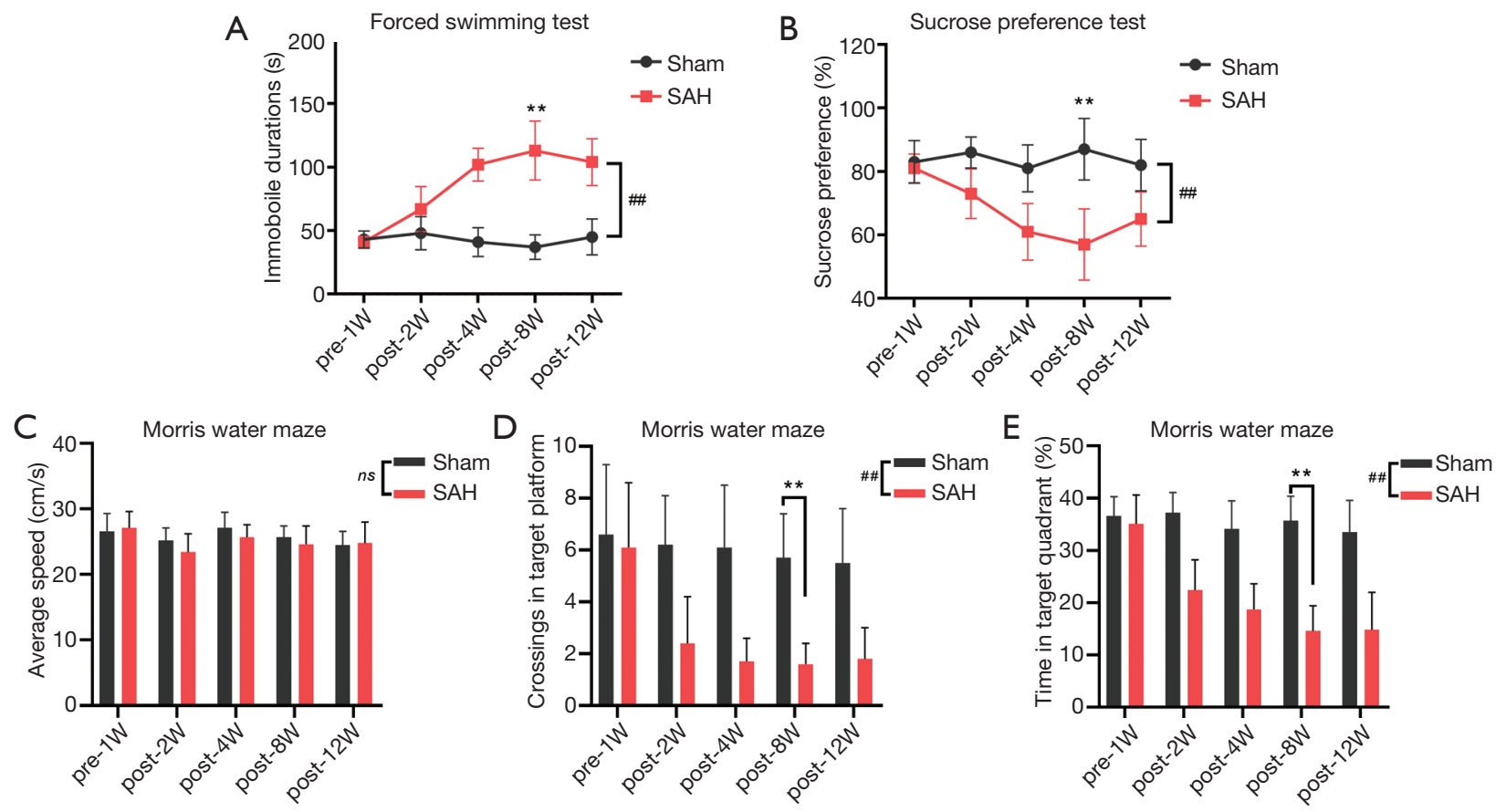

Figure 1 The DCI behavioral performance in mice after SAH. (A) Forced swimming test and (B) sugar water preference test were used to evaluate the depressive behavior of mice 1 week before SAH and 2 to 12 weeks after SAH. In the Morris water maze test, the average exercise speed (C), the crossing times of the platform area (D) and the target quadrant residence time (E) were analyzed to assess the spatial reference memory of $\mathrm{SAH}$ mice. ${ }^{\#}, \mathrm{P}<0.01$, the $\mathrm{SAH}$ group $v$ s. the sham group; ${ }^{* *}, \mathrm{P}<0.01$, vs. the indicated group. $\mathrm{N}=8$ mice at each time point of each group.

group had a significantly longer immobility time (Figure $1 \mathrm{~A}$, $\mathrm{P}<0.01)$ and a significantly lower sugar water uptake rate (Figure $1 B, \mathrm{P}<0.01$ ) than the sham group in the forced swimming and sugar preference tests, respectively. Two weeks after SAH, one-way ANOVA indicated that mice in the SAH group exhibited obvious depressive behavior. At 8 weeks after SAH, the differences in the immobility time $(113.4 \pm 22.5 \mathrm{~s} v$ s. $36.8 \pm 9.7 \mathrm{~s})$ and sugar water intake rate $(87.2 \% \pm 9.3 \%$ vs. $57.3 \% \pm 10.6 \%)$ between the $\mathrm{SAH}$ group and the sham group were the most significant $(\mathrm{P}<0.01)$; they reached a peak, and they began to decrease at 12 weeks after SAH.

As shown in Figure 1C, in the Morris water maze test, there was no significant difference in swimming speed between the sham and SAH groups at different times $(\mathrm{P}>0.05$, two-way ANOVA), suggesting that the operation in the SAH model had no significant effect on the exercise ability of the mice. In the spatial reference memory test, the number of times the SAH mice crossed the platform and the duration of time they spent in the target quadrant were significantly lower than those for the sham mice
$(\mathrm{P}<0.01$, two-way ANOVA). The differences in the number of platform crossings $(5.7 \pm 1.7 \mathrm{~s}$ vs. $1.6 \pm 0.8 \mathrm{~s}$ ) (Figure $1 D$, $\mathrm{P}<0.01)$ and the duration of time in the target quadrant $(35.6 \% \pm 4.3 \%$ vs. $14.6 \% \pm 4.5 \%$ ) (Figure $1 E, \mathrm{P}<0.01$ ) were higher in the SAH group compared with the sham group $(\mathrm{P}<0.01$, one-way ANOVA).

\section{Changes in the glutamatergic system in the hippocampus 8 weeks after $S A H$}

Glutamatergic impairment is an important mechanism in a variety of cognitive disorders. The interstitial glutamate in the hippocampus during DCI after $\mathrm{SAH}$ was measured by microdialysis and HPLC-MS/MS (Figure $2 A$ ). The glutamate concentration in the cerebrospinal fluid of the SAH group $(1,758.3 \pm 287.6 \mu \mathrm{M})$ was significantly higher than that in the sham group $(529.4 \pm 115.7 \mu \mathrm{M})$ at 8 weeks post operation, which is when the DCI was at its most severe. There was no significant difference in the sham group before and after the operation.

The interstitial glutamate in the CNS is mainly 

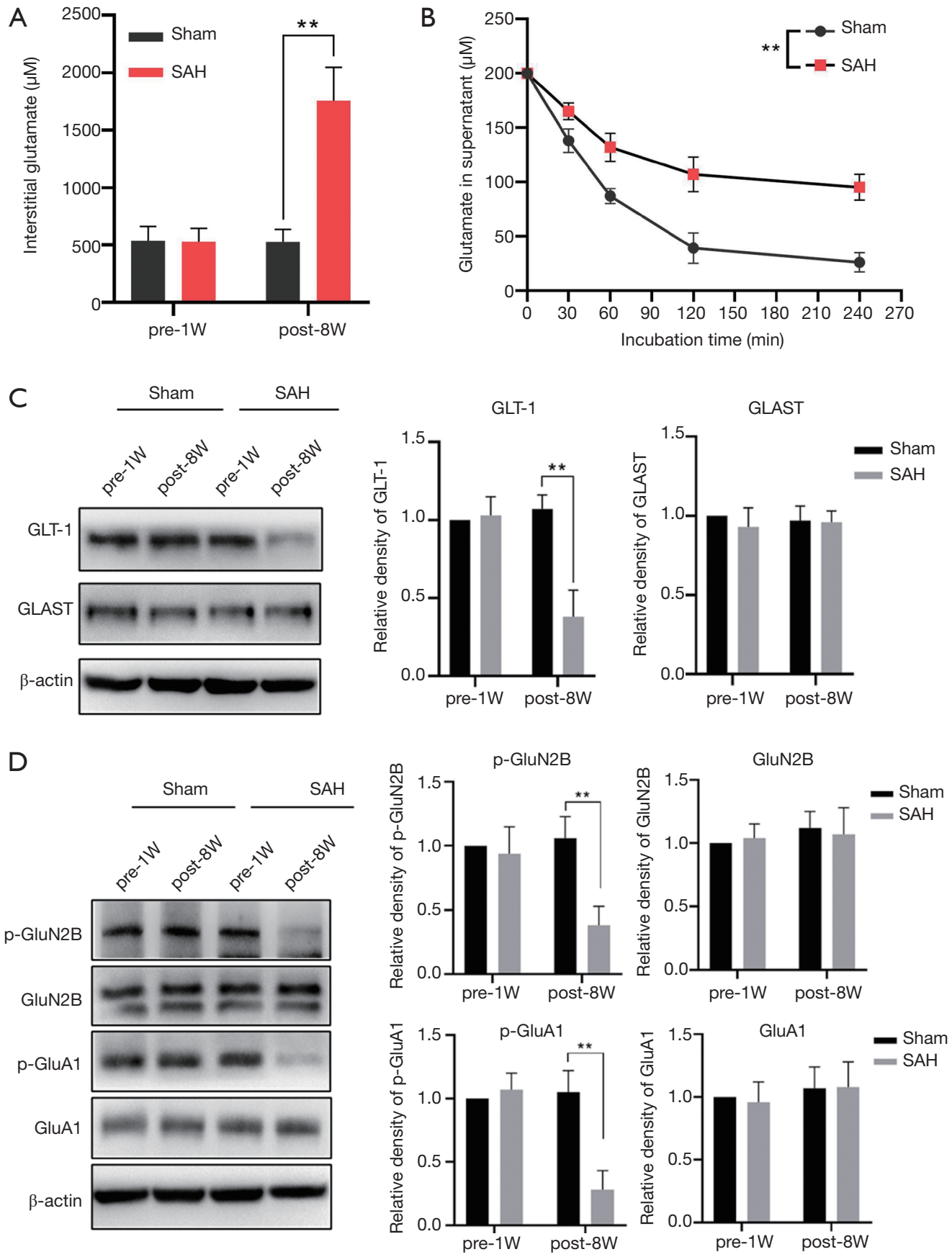

Figure 2 The changes of interstitial glutamate concentration, astrocytic glutamate reuptake function, and the expression of GluN2B, GluA1 and glutamate transporters in hippocampus after SAH at 8 weeks. (A) The concentration of interstitial glutamate in the hippocampus of the mice was detected by HPLC-MS/MS. **, $\mathrm{P}<0.01$, the $\mathrm{SAH}$ group $v$ s. the sham group. $\mathrm{N}=8$ mice per group. (B) Glutamate uptake assay on the hippocampal astrocytes sorted by immunomagnetic beads. ${ }^{* *}, \mathrm{P}<0.01$, the $\mathrm{SAH}$ group vs. the sham group. $\mathrm{N}=6$ mice per group. (C,D) Western blot was performed to detect the expression of p-GluN2B/GluN2B, p-GluA1/GluA1, GLT-1, and GLAST protein in hippocampal tissue in the sham group and the $\mathrm{SAH}$ group at 1 week before and 8 weeks after $\mathrm{SAH}$. ${ }^{* *}, \mathrm{P}<0.01$, vs. the indicated groups. $\mathrm{N}=5$ animals in each group. 
regulated by astrocytes through ATP-dependent glutamate transporters. Glutamate uptake by astrocytes during DCI after $\mathrm{SAH}$ was detected in separated and cultured astrocytes from sham and SAH mice in vitro. Glutamate concentration in the supernatant of the SAH group was significantly higher than that of the sham group $(\mathrm{P}<0.01$, two-way ANOVA) (Figure 2B).

The regulation of interstitial glutamate mainly depends on the glutamate reuptake function of EAATs on the membrane of astrocytes. Western blotting was performed to detect the expression of two subtypes of EAATs in hippocampal astrocytes (Figure 2C). The results showed that at 8 weeks after surgery, GLT-1 was significantly downregulated compared with 1 week before surgery in the SAH group $(\mathrm{P}<0.01)$, while there was no significant change in glutamate aspartate transporter (GLAST/EAAT1) $(\mathrm{P}>0.05)$. In the sham group, there was no significant difference in the expression of GLT- 1 and GLAST before and after the operation. These results suggest that the decrease in GLT-1 may be the main cause of glutamate uptake dysfunction in the hippocampus during DCI after SAH.

Abnormal accumulation of glutamate can result in a decrease in the activity of ionotropic glutamate receptors in neurons, which in turn leads to excitatory conduction disorders. After SAH, changes in glutamate receptor function in hippocampal neurons were detected by delayed cognitive impairment (DCI). The expression and phosphorylation of NMDA and AMPA receptors in the hippocampus were detected by Western blotting 8 weeks after SAH (Figure 2D). In the sham group, there were no significant differences in the levels of total protein and phosphorylation of GluN2B and GluA1 at 1 week before the operation or 8 weeks post operation. In the SAH group, there was no significant difference in the total protein expression of GluN2B and GluA1 at 8 weeks post-operation compared with the sham group $(\mathrm{P}>0.05)$, while the levels of p-GluN2B and p-GluA1 were significantly decreased $(\mathrm{P}<0.01)$. The decrease in the phosphorylation of GluN2B and GluA1 may be related to the occurrence of DCI.

\section{HDAC2 changed dramatically and negatively regulated GLT-1 expression after $S A H$}

The primary astrocytes in the hippocampus of SAH mice were sorted by immunomagnetic beads at different time points, and the expression of different subtypes of class I and II HDACs were detected by Western blotting (Figure $3 A$ ). Among the class I HDACs, HDAC2 was increased most significantly at 2-12 weeks after SAH compared with 1 week before $\mathrm{SAH}$, and HDAC2 expression was more than 2 times higher than that before SAH $(\mathrm{P}<0.01)$; HDAC1 revealed no significant changes at 2 week group but was slightly increased in the post-12 week group compared to that in the pre- $1 \mathrm{w}$ group $(\mathrm{P}<0.05,0.28 \pm 0.17$-fold changed $)$. HDAC 3 was increased slightly at 8 to 12 weeks post operation $(\mathrm{P}<0.05)$. Among the class II HDACs, HDAC4 was only increased significantly at 12 weeks after $\mathrm{SAH}$ (1.45 \pm 0.13 -fold changed compared to the pre- $1 \mathrm{w}$ group). There was no significant change in HDAC5 at any time point after SAH, and HDAC6 was decreased significantly at 2 weeks after $\mathrm{SAH}(\mathrm{P}<0.05,0.41 \pm 0.11$ fold of the pre- $1 \mathrm{w}$ group), but was increased at 8 and 12 weeks after surgery $(\mathrm{P}<0.05$, respectively).

In order to further determine the significant changes of HDAC2 expression in astrocytes before and after SAH, which may affect the expression of GLT-1, we performed immunofluorescence staining to triple-label GFAP, HDAC2 and GLT-1 in astrocytes. The results showed that astrocytes were significant activation at 8 weeks after SAH (Figure 3B). Co-location showed that HDAC2 expression was significantly increased in astrocytes of SAH group, while GLT-1 expression was significantly decreased. These results indicated that the alteration of HDAC2 in astrocytes was most significant during DCI after SAH, which may be related to the downregulation of GLT-1.

The effect of HDAC2 on GLT-1 expression after $\mathrm{SAH}$ was further investigated. GLT-1 expression in the hippocampus was detected at 8 weeks after SAH following 6 weeks of treatment with an HDAC2-specific inhibitor (HDACi, santacruzamate A). The results (Figure 3C,D) showed that the expressions of hippocampal GLT-1 protein $(\mathrm{P}<0.05)$ and mRNA $(\mathrm{P}<0.05)$ were significantly increased by the additional HDAC2i treatment in the SAH mice compared with those in the vehicle-treated mice.

\section{Pharmacological inbibition of HDAC2 alleviated DCI after SAH-dependent GLT-1 activity}

Further exploration was performed to detect the effect of HDAC2 inhibition on DCI after SAH in mice. For 6 weeks, SAH mice were intraperitoneally treated with the HDAC2specific inhibitor santacruzamate alone or in combination with the selective GLT-1 inhibitor WAY-213613. Western blot showed that the expressions of GLT-1, p-GluN2B and p-GluA1 in hippocampus were decreased, while HDAC2 was increased after SAH. Treatment of HDAC2 inhibitor 

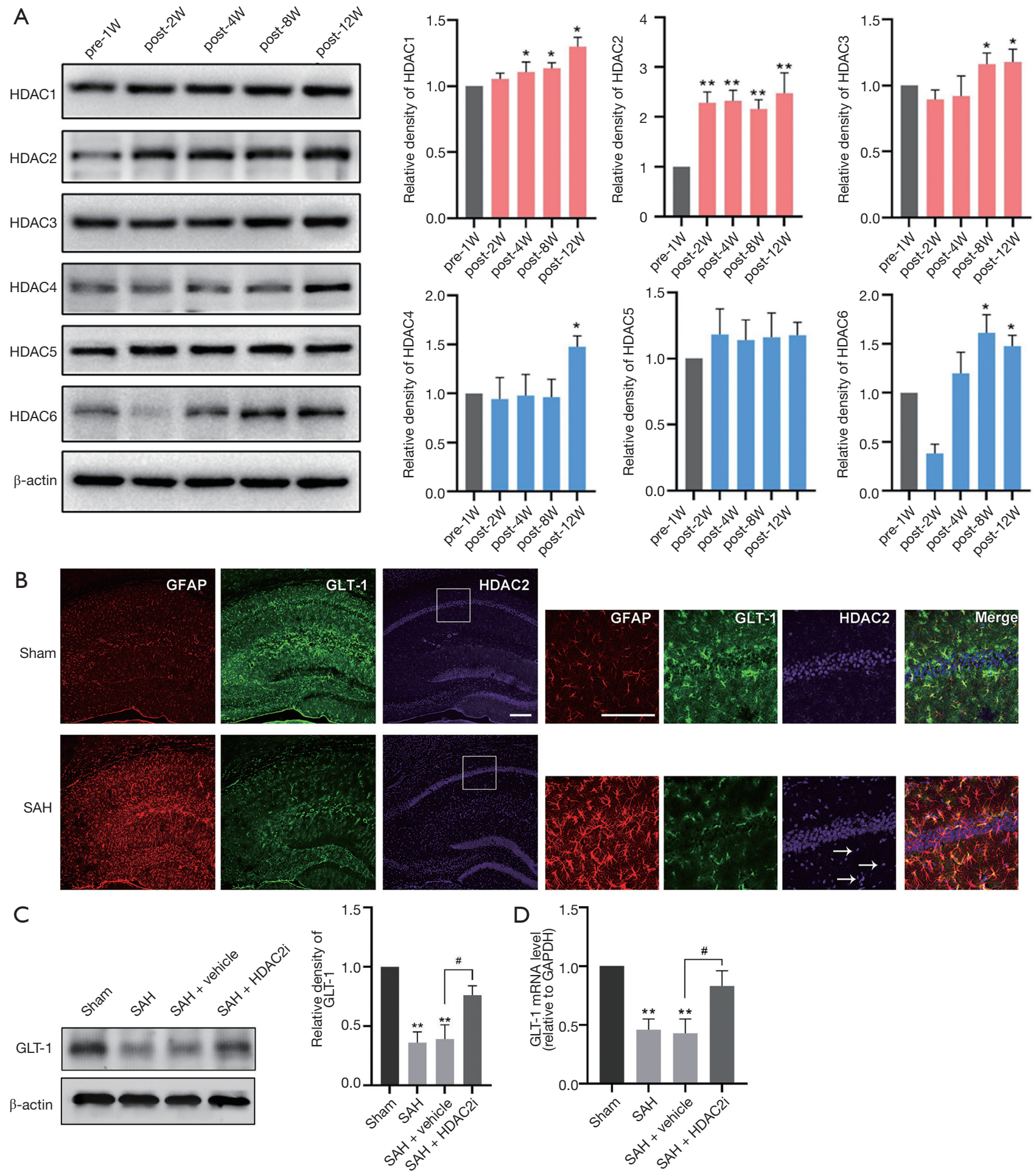

Figure 3 The expression of different HDACs in the hippocampal astrocytes of SAH mice after SAH and the effect of HDAC2 inhibition on GLT-1 expression. (A) Western blot detected the expression of different subtypes of class I and II HDACs in hippocampal astrocytes sorted from SAH mice. * $\mathrm{P}<0.05$, **, $\mathrm{P}<0.01$, vs. the pre-1 week group. $\mathrm{N}=5$ mice per group. (B) The co-localization of GLT-1, HDAC2 and GFAP was detected by immunofluorescence staining. Scale bar $=100 \mu \mathrm{m}$, zoom in $=50 \mu \mathrm{m}$. $\mathrm{N}=5$ animals in each group. The protein (C) and mRNA (D) level of GLT-1 in the hippocampus of the mice. **, $\mathrm{P}<0.01$, vs. the sham group; ${ }^{*}, \mathrm{P}<0.05$, vs. the indicated groups. $\mathrm{N}=5$ mice per group. 
increased the expressions of GLT-1, p-GluN2B and p-GluA1 compared to the SAH group, while p-GluN2B and p-GluA1 were decreased when combined administration of HDAC2 and GLT-1 inhibitors (Figure 4A). Since the inhibitors of HDAC2 and GLT-1 only inhibit the functional activity of proteins, the expressions of these proteins showed no significant change.

In the forced swimming test and sugar preference test, the immobility time was significantly decreased $(\mathrm{P}<0.01$, $v s$. the $\mathrm{SAH}$ group) (Figure $4 B$ ), and the sugar water uptake rate was significantly increased $(\mathrm{P}<0.05$, vs. the $\mathrm{SAH}$ group) (Figure $4 C$ ) in SAH mice after treatment with the HDAC2 inhibitor, which indicated that HDAC2 inhibition could effectively alleviate depression in SAH mice. In the water maze test, $\mathrm{SAH}$ induced obvious dysfunction of spatial learning memory (Figure $4 D, \mathrm{P}<0.01$ ) and spatial reference memory (Figure $4 E, \mathrm{P}<0.01$ ) compared to the sham treatment. Treatment with the HDAC2 inhibitor significantly reduced the escape latency $(\mathrm{P}<0.01$, vs. the $\mathrm{SAH}$ group) and increased the duration in the target quadrant of $\mathrm{SAH}$ mice $(\mathrm{P}<0.05$, vs. the $\mathrm{SAH}$ group), suggesting that HDAC2 inhibition could effectively reduce the memory impairment of SAH mice. However, both the antidepressant and learning and memory improvement effects of HDAC2 inhibitors were markedly reduced when they were combined with a GLT- 1 inhibitor (Figure 4B,C,D,E, $\mathrm{P}<0.05$, SAH + HDAC2i + WAY group vs. $\mathrm{SAH}+\mathrm{HDAC} 2 \mathrm{i}$ group). These results suggested that the effect of HDAC2 inhibition on the improvement of cognitive function in SAH mice may depend on the activation of GLT-1 in astrocytes.

\section{Discussion}

DCI after SAH is one of the most common sequelae in clinical practice, which seriously impacts the life quality of SAH patients $(18,19)$. The pathogenesis of DCI after SAH is still unknown. In this study, we used SAH mouse model to explore the characteristics of DCI after SAH and the possible mechanism of "glutamatergic disorder" $(20,21)$. The main findings were as follows: (I) delayed depression and memory disorder can persist in SAH mice for 12 weeks, with the most severe manifestations observed at 8 weeks; (II) downregulation of GLT-1 but not of GLAST on astrocytes may result in the accumulation of glutamate in the hippocampus and neurons after SAH induction, which is the chief cause of glutamate dysfunction in astrocytes; (III) transcriptional inhibition of GLT-1 expression by
HDAC2 is an epigenetic mechanism of astrocyte GLT-1 downregulation after SAH; and (IV) specific inhibition of HDAC2 may improve DCI after SAH via GLT-1.

Clinically, cognitive dysfunction in SAH patients can occur 1 week after the onset of SAH and last for more than 6 months (22-24). To reduce the effect of wound pain on the behavior of the mice in this study, we began behavioral testing after 2 weeks of internal carotid puncture. The results showed that the SAH mice developed significant depression and memory impairment within 2 weeks compared with the sham operated mice, which suggests that cognitive dysfunction after SAH may occur less than 2 weeks after onset and that the onset time may be consistent with that observed in clinical patients (23). The most severe depression and memory impairment were observed at 8 weeks after $\mathrm{SAH}$, which started to reduce from 12 weeks. To some extent, the DCI behaviors of this SAH mouse model reflect those of SAH patients.

Clinical imaging and pathological studies have confirmed that hippocampal atrophy and neurotransmitter abnormalities in SAH patients are related to the severity of depression and anxiety (25). Among the disordered transmitters, glutamate accumulation was a major cause of NMDAR and AMPAR dysfunction in a variety of pathological states $(26,27)$, which impaired the synaptic plasticity of hippocampal neurons $(28,29)$, and induced cognitive dysfunction. GLT-1 is mainly expressed on hippocampal astrocytes (30), and we found that GLT1 expression was significantly downregulated during the onset of DCI after SAH, while GLAST expression did not significantly change, which is consistent with the previous findings in SAH rat model (31). These results further suggest that reduced GLT-1 expression on astrocytes may be an important mechanism of DCI after SAH.

The downregulation of GLT-1 expression after SAH was confirmed in our previous study to mainly be caused by transcriptional suppression. As well as the involvement of transcription factors such as CREB and NF- $\mathrm{KB}$ in GLT-1 regulation, histone acetylation plays a role that cannot be ignored (32). Multiple studies have confirmed that GLT-1 expression can be upregulated by the broad-spectrum inhibitors of HDAC (11). Consequently, identifying the key HDAC subtypes that affect the expression of GLT-1 in astrocytes after SAH and performing these specific inhibitory interventions may reduce the serious side effects caused by those broad-spectrum HDAC inhibitors. In the current study, we detected the expression changes of type I and type II HDACs in the hippocampus during the 

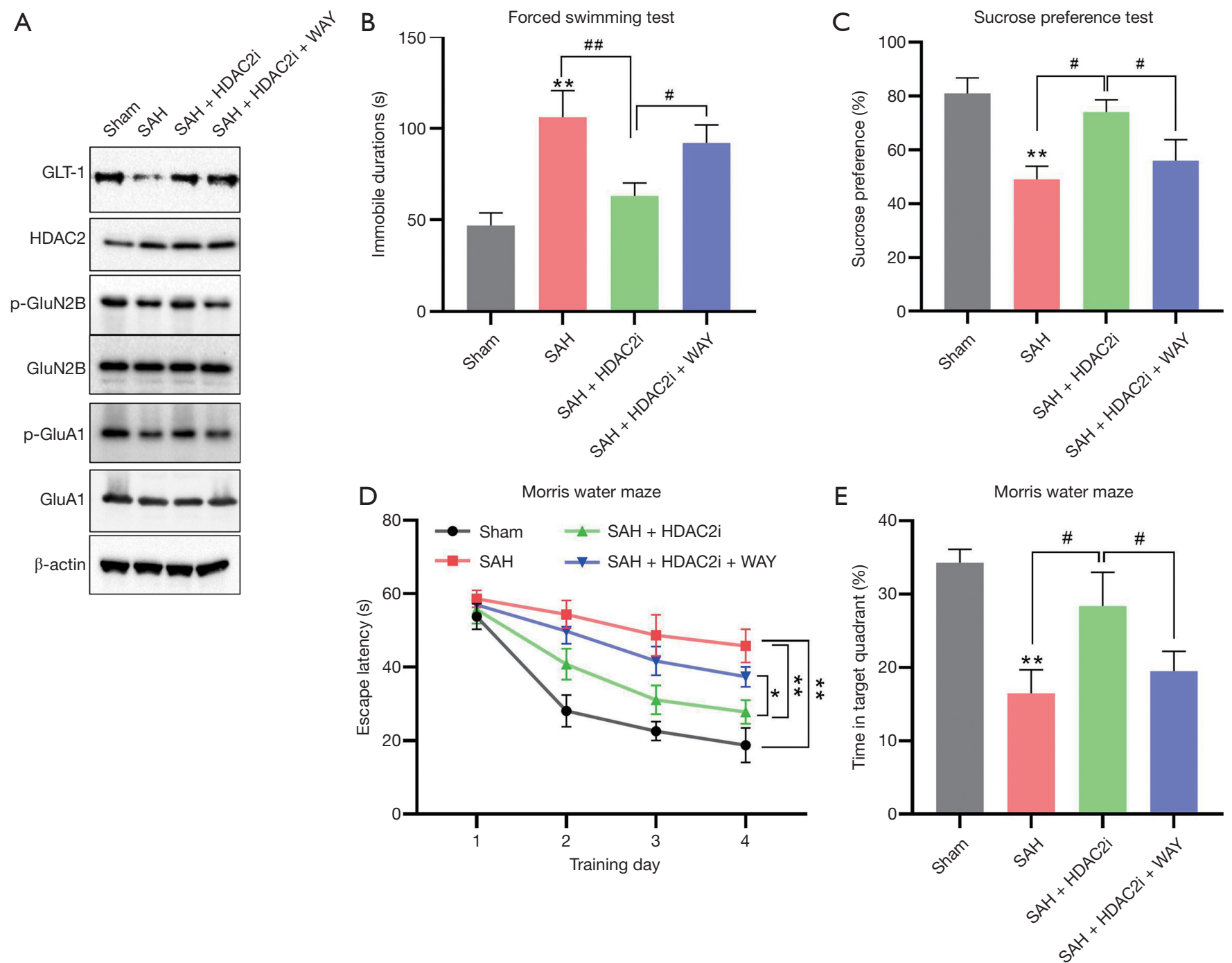

Figure 4 Effects of selective HDAC2 inhibitor and GLT-1 inhibitor on DCI in SAH mice. (A) The SAH mice were intraperitoneally administered with HDAC2 inhibitor $(2 \mathrm{mg} / \mathrm{kg}$ ) and GLT-1 inhibitor (WAY-213613, $1 \mathrm{mg} / \mathrm{kg}$ ) two weeks after surgery once every other day. The expression of GLT-1, HDAC2, p-GluN2B and p-GluA1 in hippocampus was detected after SAH at 8 weeks. N=5 animals in each group. (B) Forced swimming test and (C) sugar water preference test were used to evaluate the depressive behavior of SAH mice treated with HDAC2 inhibitor (Santacruzamate A) and GLT-1 inhibitor (WAY-213613). **, $\mathrm{P}<0.01$, vs. the sham group; ${ }^{\#}, \mathrm{P}<0.05$ and ${ }^{\# \#}, \mathrm{P}<0.01, v s$. the indicated groups. (D) Morris water maze test was used to detect the escape latency for reflecting spatial learning memory, * $\mathrm{P}<0.01$, and **, $\mathrm{P}<0.01$, vs. the indicated groups. (E) Reference memory was detected by recording the time in target quadrant of Morris water maze test. **, $\mathrm{P}<0.01$, vs. the sham group; ${ }^{\#}, \mathrm{P}<0.05$, vs. the indicated groups. $\mathrm{N}=8$ mice per group.

onset of DCI after SAH. For the first time, we identified HDAC2 as being closely related to abnormal GLT-1 expression in astrocytes after SAH. Santocruzamate A, the selective inhibitor of HDAC2, which possesses an IC50 of $119 \mathrm{pM}$ for HDAC2, is 3,600 times higher than other subtypes of HDACs $(33,34)$. Santacruzamate A treatment effectively rescued the reduction of GLT-1 expression in the hippocampus after $\mathrm{SAH}$, which provides a basis for this intervention of DCI in animals. Furthermore, previous reports have indicated that in response to different pathological stimuli, GLT-1 undergoes different post-translational modifications (PTMs), including ubiquitination, sumoylation, and phosphorylation, all of which affect protein stability and lead to protein 


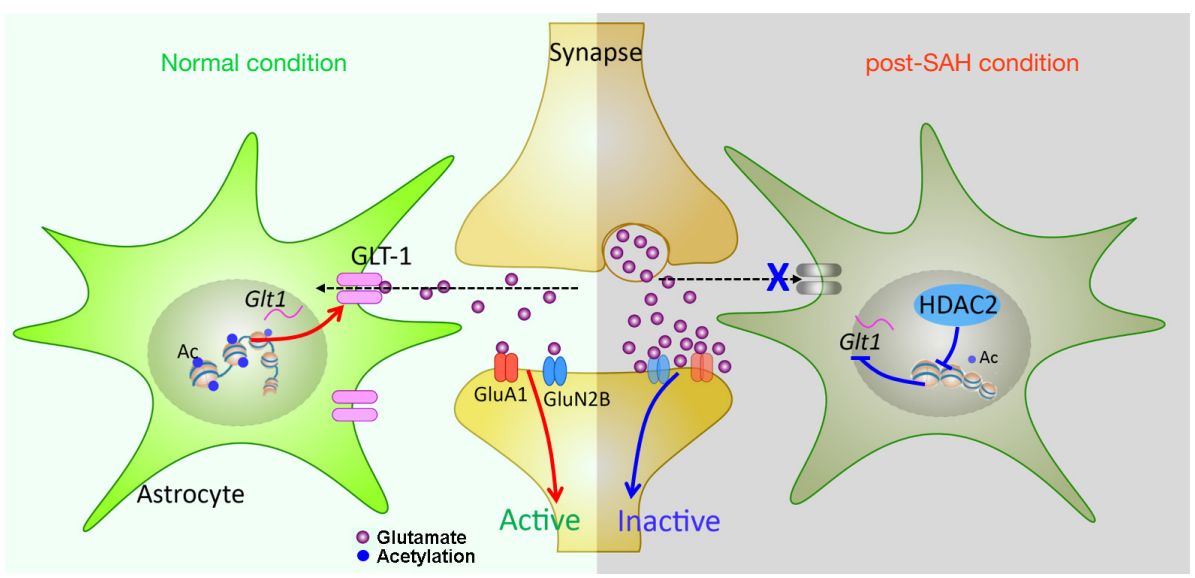

Figure 5 Negative regulation of GLT-1 by astrocytes HDAC2 leads to dysfunction of glutamate reuptake in the synaptic cleft. In normal condition, the acetylation of histones in astrocytes facilitates the transcriptional regulation of GLT-1. Glutamate in the synaptic cleft is rapidly absorbed into astrocytes to maintain excitability of synapses. The increase of HDAC2 in astrocytes after SAH results in the deacetylation of histones and inhibits the transcription expression of GLT-1. The decrease of GLT-1 expression will lead to the impairment of glutamate reuptake in astrocytes and the long-term accumulation of glutamate in the synaptic space, resulting the dephosphorylation of ionized glutamate receptors GluN2B and GluA1 on the postsynaptic membrane. These eventually result in the long-term inhibition of synaptic excitability and DCI. DCI, delayed cognitive impairment.

degradation (35-37). However, whether PTMs occur in GLT-1 after SAH has not yet been reported and is an area worthy of further study.

Histone acetylation inhibitors have been reported to affect memory function. Previous studies have confirmed that the HDAC inhibitor SAHA enhanced memory formation, and its effect depended on the inhibition of broad-spectrum HDACs (38). HDAC2, in particular, negatively regulated hippocampal synaptogenesis and synaptic plasticity by inhibiting neuronal gene expression $(39,40)$. In this study, we found that HDAC2 was also involved in the regulation of gene expression in astrocytes after SAH. Despite this study not specifically detecting the changes of HDAC2 in neurons after SAH and its role in DCI, the expression of GLT-1 in the hippocampal astrocytes of SAH mice significantly recovered after treatment with specific HDAC2 inhibition. At the same time, behavioral pharmacological experiments also confirmed that the effect of HDAC2 inhibition on alleviating DCI after SAH depended in part on the activity of astrocytic GLT-1. Therefore, at least partially, our results suggested that the HDAC2-GLT-1 pathway in astrocytes plays an important role in the regulation of DCI after SAH.

In summary, we confirmed the course characteristics of delayed depression and memory impairment in SAH mouse model. The role of astrocytic GLT-1-mediated glutamate homeostasis in the occurrence of DCI after SAH, as well as the mechanism of HDAC2-mediated GLT-1 transcriptional regulation, were discovered (Figure 5). Our study also suggested that inhibitors targeting HDAC2 may serve as a potential treatment for cognitive dysfunction after $\mathrm{SAH}$.

\section{Acknowledgments}

Funding: This project was supported by National Natural Science Foundation of China (grant No. 81971129 and No. 81500909 to DF) and National Natural Science Foundation of Shaanxi province (grant No. 2018JM7019).

\section{Footnote}

Reporting Checklist: The authors have completed the ARRIVE reporting checklist. Available at http://dx.doi. org/10.21037/atm-20-4330

Data Sharing Statement: Available at http://dx.doi. org/10.21037/atm-20-4330

Conflicts of Interest: All authors have completed the ICMJE uniform disclosure form (available at http://dx.doi. org/10.21037/atm-20-4330). LL serves as an unpaid editorial board member of Annals of Translational Medicine 
from Apr 2020 to Mar 2022. The other authors have no conflicts of interest to declare.

Ethical Statement: The authors are accountable for all aspects of the work in ensuring that questions related to the accuracy or integrity of any part of the work are appropriately investigated and resolved. All procedures were approved by the Institutional Animal Care and Use Committee and performed in accordance with the ethical guidelines of the Fourth Military Medical University (animal approval number: TDLL-2015034). The operations were performed to minimize the number and suffering of animals used.

Open Access Statement: This is an Open Access article distributed in accordance with the Creative Commons Attribution-NonCommercial-NoDerivs 4.0 International License (CC BY-NC-ND 4.0), which permits the noncommercial replication and distribution of the article with the strict proviso that no changes or edits are made and the original work is properly cited (including links to both the formal publication through the relevant DOI and the license). See: https://creativecommons.org/licenses/by-nc-nd/4.0/.

\section{References}

1. Gu HQ, Yang X, Rao ZZ, et al. Disparities in outcomes associated with rural-urban insurance status in China among inpatient women with stroke: a registry-based cohort study. Ann Transl Med 2019;7:426.

2. Reijmer YD, van den Heerik MS, Heinen R, et al. Microstructural White Matter Abnormalities and Cognitive Impairment After Aneurysmal Subarachnoid Hemorrhage. Stroke 2018;49:2040-5.

3. Burke T, Hughes S, Carr A, Javadpour M, Pender N. A Systematic Review of Cognitive Outcomes in Angiographically Negative Subarachnoid Haemorrhage. Neuropsychol Rev 2018;28:453-69.

4. Ma S, Hangya B, Leonard CS, et al. Dual-transmitter systems regulating arousal, attention, learning and memory. Neurosci Biobehav Rev 2018;85:21-33.

5. Saveland H, Nilsson OG, Boris-Moller F, et al. Intracerebral microdialysis of glutamate and aspartate in two vascular territories after aneurysmal subarachnoid hemorrhage. Neurosurgery 1996;38:12-9; discussion 19-20.

6. Castillo J, Davalos A, Noya M. Progression of ischaemic stroke and excitotoxic aminoacids. Lancet 1997;349:79-83.

7. Sha L, Wang X, Li J, et al. Pharmacologic inhibition of Hsp90 to prevent GLT-1 degradation as an effective therapy for epilepsy. J Exp Med 2017;214:547-63.

8. Lin BF, Kuo CY, Wen LL, et al. Rosiglitazone attenuates cerebral vasospasm and provides neuroprotection in an experimental rat model of subarachnoid hemorrhage. Neurocrit Care 2014;21:316-31.

9. Hoshi A, Tsunoda A, Yamamoto T, Tada M, Kakita A, Ugawa Y. Altered expression of glutamate transporter-1 and water channel protein aquaporin- 4 in human temporal cortex with Alzheimer's disease. Neuropathol Appl Neurobiol 2018;44:628-38.

10. Ghosh M, Yang Y, Rothstein JD, Robinson MB. Nuclear factor-kappaB contributes to neuron-dependent induction of glutamate transporter-1 expression in astrocytes. J Neurosci 2011;31:9159-69.

11. Itoh $M$, Hiroi T, Nishibori N, et al. Trichostatin A enhances glutamate transporter GLT-1 mRNA levels in C6 glioma cells via neurosteroid-mediated cell differentiation. J Mol Neurosci 2013;49:21-7.

12. Suzuki H, Sozen T, Hasegawa Y, Chen W, Zhang JH. Caspase-1 inhibitor prevents neurogenic pulmonary edema after subarachnoid hemorrhage in mice. Stroke 2009;40:3872-5.

13. Dong $\mathrm{Y}, \mathrm{Zhou} \mathrm{Y}, \mathrm{Chu} \mathrm{X}$, et al. Dental noise exposed mice display depressive-like phenotypes. Mol Brain 2016;9:50.

14. Sasibhushana RB, Shankaranarayana RB, Srikumar BN. Repeated finasteride administration induces depressionlike behavior in adult male rats. Behav Brain Res 2019;365:185-9.

15. Li Y, Yin A, Sun X, et al. Deficiency of tumor suppressor NDRG2 leads to attention deficit and hyperactive behavior. J Clin Invest 2017;127:4270-84.

16. Zhou J, Tao K, Guo K, et al. Suppression of NDRG2 alleviates brain injury after intracerebral hemorrhage through mitigating astrocyte-drived glutamate neurotoxicity via NF-kappaB/GLT1 signaling. Brain Res 2020;1729:146600.

17. Han J, Pedersen JS, Kwon SC, et al. Posttranscriptional Crossregulation between Drosha and DGCR8. Cell 2009;136:75-84.

18. James RF, Khattar NK, Aljuboori ZS, et al. Continuous infusion of low-dose unfractionated heparin after aneurysmal subarachnoid hemorrhage: a preliminary study of cognitive outcomes. J Neurosurg 2018:1-8.

19. Persson HC, Tornbom M, Winso O, Sunnerhagen KS. Symptoms and consequences of subarachnoid haemorrhage after 7 years. Acta Neurol Scand 2019;140:429-34.

20. Tu CH, MacDonald I, Chen YH. The Effects of Acupuncture on Glutamatergic Neurotransmission in 
Depression, Anxiety, Schizophrenia, and Alzheimer's Disease: A Review of the Literature. Front Psychiatry 2019;10:14.

21. Moretto E, Murru L, Martano G, Sassone J, Passafaro M. Glutamatergic synapses in neurodevelopmental disorders. Prog Neuropsychopharmacol Biol Psychiatry 2018;84:328-42.

22. Tolli A, Hoybye C, Bellander BM, Johansson F, Borg J. The effect of time on cognitive impairments after nontraumatic subarachnoid haemorrhage and after traumatic brain injury. Brain Inj 2018;32:1465-76.

23. Eagles ME, Tso MK, Macdonald RL. Cognitive Impairment, Functional Outcome, and Delayed Cerebral Ischemia After Aneurysmal Subarachnoid Hemorrhage. World Neurosurg 2019. [Epub ahead of print].

24. Provencio JJ, Swank V, Lu H, et al. Neutrophil depletion after subarachnoid hemorrhage improves memory via NMDA receptors. Brain Behav Immun 2016;54:233-42.

25. Wostrack M, Friedrich B, Hammer K, et al. Hippocampal damage and affective disorders after treatment of cerebral aneurysms. J Neurol 2014;261:2128-35.

26. Xhima K, Weber-Adrian D, Silburt J. Glutamate Induces Blood-Brain Barrier Permeability through Activation of N-Methyl-D-Aspartate Receptors. J Neurosci 2016;36:12296-8.

27. Papazian I, Kyrargyri V, Evangelidou M, et al. Mesenchymal Stem Cell Protection of Neurons against Glutamate Excitotoxicity Involves Reduction of NMDATriggered Calcium Responses and Surface GluR1, and Is Partly Mediated by TNF. Int J Mol Sci 2018;19:651.

28. Yu D, Cheng Z, Ali AI, et al. Chronic Unexpected Mild Stress Destroys Synaptic Plasticity of Neurons through a Glutamate Transporter, GLT-1, of Astrocytes in the Ischemic Stroke Rat. Neural Plast 2019;2019:1615925.

29. Zhou Z, Liu A, Xia S, et al. The C-terminal tails of endogenous GluA1 and GluA2 differentially contribute to hippocampal synaptic plasticity and learning. Nat Neurosci 2018;21:50-62.

30. Huang YH, Sinha SR, Tanaka K, et al. Astrocyte glutamate transporters regulate metabotropic glutamate receptormediated excitation of hippocampal interneurons. J Neurosci 2004;24:4551-9.

31. Kuo CP, Wen LL, Chen CM, et al. Attenuation of neurological injury with early baicalein treatment following subarachnoid hemorrhage in rats. J Neurosurg 2013;119:1028-37.
32. Perisic T, Zimmermann N, Kirmeier T, et al. Valproate and amitriptyline exert common and divergent influences on global and gene promoter-specific chromatin modifications in rat primary astrocytes. Neuropsychopharmacology 2010;35:792-805.

33. Pavlik CM, Wong CY, Ononye S, et al. Santacruzamate A, a potent and selective histone deacetylase inhibitor from the Panamanian marine cyanobacterium cf. Symploca sp. J Nat Prod 2013;76:2026-33.

34. Zhou H, Cai Y, Liu D, et al. Pharmacological or transcriptional inhibition of both HDAC1 and 2 leads to cell cycle blockage and apoptosis via p21(Waf1/Cip1) and p19(INK4d) upregulation in hepatocellular carcinoma. Cell Prolif 2018;51:e12447.

35. Garcia-Tardon N, Gonzalez-Gonzalez IM, MartinezVillarreal J, et al. Protein kinase C (PKC)-promoted endocytosis of glutamate transporter GLT-1 requires ubiquitin ligase Nedd4-2-dependent ubiquitination but not phosphorylation. J Biol Chem 2012;287:19177-87.

36. Chen T, Tanaka M, Wang Y, et al. Neurosteroid dehydroepiandrosterone enhances activity and trafficking of astrocytic GLT-1 via sigma1 receptor-mediated PKC activation in the hippocampal dentate gyrus of rats. Glia 2017;65:1491-503.

37. Foran E, Rosenblum L, Bogush A, et al. Sumoylation of the astroglial glutamate transporter EAAT2 governs its intracellular compartmentalization. Glia 2014;62:1241-53.

38. Sartor GC, Malvezzi AM, Kumar A, et al. Enhancement of BDNF Expression and Memory by HDAC Inhibition Requires BET Bromodomain Reader Proteins. J Neurosci 2019;39:612-26.

39. Tang Y, Li MY, Zhang X, et al. Delayed exposure to environmental enrichment improves functional outcome after stroke. J Pharmacol Sci 2019;140:137-43.

40. Guan JS, Haggarty SJ, Giacometti E, et al. HDAC2 negatively regulates memory formation and synaptic plasticity. Nature 2009;459:55-60.

Cite this article as: Tao K, Cai Q, Zhang X, Zhu L, Liu Z, Li F, Wang Q, Liu L, Feng D. Astrocytic histone deacetylase 2 facilitates delayed depression and memory impairment after subarachnoid hemorrhage by negatively regulating glutamate transporter-1. Ann Transl Med 2020;8(11):691. doi: 10.21037/ atm-20-4330 